\title{
A switch in task affects priming of pop-out: evidence for the role of episodes
}

\author{
David R. Thomson • Bruce Milliken
}

Published online: 16 November 2010

(C) Psychonomic Society, Inc. 2010

\begin{abstract}
Maljkovic and Nakayama (Memory \& Cognition, 22(6), 655-678, 1994) demonstrated that response times decrease in a pop-out search task when target-defining features repeat from one trial to the next. This priming of pop-out (PoP) effect has been explained by some researchers as reflecting low-level modulations in attentional control settings Lee, Mozer, and Vecera (Attention, Perception, \& Psychophysics, 71(5), 1059-1071, 2009). The present experiments tested whether a shift in higher order task requirements from trial $n-1$ to trial $n$ alters PoP effects. The results of Experiments 1 and 2 demonstrated that a switch in task significantly modulated PoP effects when shape was the relevant pop-out dimension. Experiment 3 failed to show significant modulation of PoP as a function of task switch when the pop-out dimension was color, but the findings of Experiment 4 did show modulation of PoP for color when the relative salience of target and distractors was high. Together, the results strongly support the view that PoP effects can be sensitive to a switch in task, a result consistent with the view that PoP effects are modulated by trial-to-trial episodic integration processes.
\end{abstract}

Keywords Priming · Singleton search · Implicit memory . Attention

\section{Introduction}

The way in which we select relevant information from a cluttered visual scene has been the subject of considerable

\section{R. Thomson $(\bowtie) \cdot$ B. Milliken}

Department of Psychology, Neuroscience \& Behavior,

McMaster University,

Hamilton, ON, Canada

e-mail: thomsodr@mcmaster.ca study in cognitive psychology. A task often used for this purpose is visual search. It is a well-known finding in these studies that some types of search are more efficient than others. For example, when searching for a conjunctively defined target amongst heterogeneous distractors, search times are particularly slow and tend to increase as the number of distracting items in the display increases. On the other hand, when searching for a singleton target amongst homogeneous distractors, search has been shown to be particularly fast and far less sensitive to manipulations of set size (Treisman \& Gelade, 1980). This latter observation has been termed popout, to capture the idea that the target pops out from the field of distractors without requiring an item-by-item search.

Pop-out in visual search has often been attributed to lowlevel bottom-up processes that do not require attention (Treisman \& Gelade, 1980). According to this view, the efficient detection of a pop-out target relies on a salience map that serves to draw attention to areas in space that are particularly salient with respect to surrounding perceptual information. In accord with this idea, localization of a popout target has been described as "pre-attentive" (Treisman \& Gelade, 1980).

A role for memory in pop-out search was demonstrated, however, by Maljkovic and Nakayama (1994). They asked participants to search for an odd-colored singleton target in the presence of two homogeneous distracting items, and to indicate whether the left or right corner of the target diamond was missing. They demonstrated that responses were faster when the same colored target repeated from one trial to the next than when the target color switched from one trial to the next. Maljkovic and Nakayama called this effect priming of pop-out (PoP), and they noted that it implied that pop-out search is mediated not just by the bottom-up salience map created by the perceptual properties of the current stimulus display but is also mediated by 
the past experiences of the observer (Maljkovic \& Nakayama, 1994, 1996, 2000). The mechanisms underlying PoP have since been the topic of considerable study, a brief overview of which follows.

PoP effects have been described by some researchers as reflecting the operation of a memory trace that encodes target-defining feature information only. The simple idea here is that memory for the previous target-defining feature can facilitate search if the target in a subsequent display matches the contents of that memory trace (Bravo \& Nakayama, 1992; Maljkovic \& Nakayama, 1994, 1996). According to this view, this memory trace is implicit in nature, as subjects appear to lack awareness of target and distracting properties of preceding trials (Maljkovic \& Nakayama, 2000), and is relatively short term, as it exerts an influence on search performance for 5-8 subsequent trials (Maljkovic \& Nakayama, 1994, experiment 5). Maljkovic and Nakayama (1994) also demonstrated that these priming effects are not driven by response repetition effects from trial to trial (their experiment 6) and do not depend on the expectations of the observer (their experiment 2), and so concluded that the implicit memory trace for target-defining features exerts its influence at a pre-attentive stage in search, as described by Treisman and Gelade (1980).

While some researchers have gathered additional empirical support for the notion of a memory influence that is specific to target-defining information (Hillstrom, 2000), others have noted that target-irrelevant information can also have an impact on search performance for pop-out targets (Huang, Holcombe, \& Pashler, 2004; Kristjansson, 2006; McBride, Leonards, \& Gilchrist, 2009; Olivers \& Humphreys, 2003). Huang et al. (2004) had subjects perform a pop-out search task for an odd-sized target, in which they had to report the orientation of the odd-sized singleton. The color of the target was irrelevant to the task; nonetheless, results showed that search times for repeated targets on the task-relevant dimension (size) were further expedited if the task-irrelevant dimension (color) also repeated from one trial to the next. Interestingly, a switch in the target-defining dimension across trials was further slowed by a match in the target-irrelevant dimension. These results seem to indicate that more than target-defining feature information affects pop-out search performance. More recently, Lee, Mozer, and Vecera (2009), using a procedure similar to Maljkovic and Nakayama (1994), had participants search for an odd-colored target item amongst a set of homogeneous distractors. In one experiment, an irrelevant task was inserted inbetween search displays. The irrelevant task required participants to indicate which end of a shape presented in isolation was pointed (top or bottom). Crucially, results showed that if the color of the irrelevant shape matched the color of the singleton target on a subsequent search display, search was facilitated. Taken together, the results of these studies require a reconsideration of the mechanisms that mediate pop-out search performance from trial to trial. That is, a theory must be invoked that takes into account more than just target-defining features.

An alternative view that accommodates influences beyond those of target-defining features assumes that PoP effects are best explained by reference to both low-level feature priming and trial-to-trial adjustments in cognitive control settings (Lee et al., 2009; Wolfe, 2003a, 2003b; Wolfe, Butcher, Lee, \& Hoyle, 2003). By this account, as attention is drawn to the area of the salience map with the highest activation in the visual scene, there is a relative activation of the feature dimension that defines the target (for example, 'red' gets activated relative to 'green' if the target and distractors are red and green, respectively). Devoting attention to this taskrelevant feature information necessarily means that other information bound up in the target object may also be encoded (such as task-irrelevant target shape in a color singleton task). Features that are activated by the target of the current stimulus display are assigned relative weights based on task-relevance, such that the target-defining feature will guide future responding more so than the task-irrelevant feature. This feature priming account of PoP effects posits independent activation and suppression of low-level target and distractor information (Lee et al., 2009; Olivers \& Humphreys, 2003). Although the feature priming process is mediated by cognitive control processes, it is related to the original view of Maljkovic and Nakayama in that low-level feature priming processes remain the predominant mechanism responsible for PoP.

A very different theoretical account of PoP assumes that it is best explained not by reference to low-level feature priming but instead by reference to the operation of episodic memory representations that are created and retrieved on each trial. The study by Huang et al. (2004) described earlier demonstrates an interaction between target-relevant and target-irrelevant feature repetitions from trial to trial. The fact that a repetition of the target-irrelevant color dimension can speed responding on some trials and slow responding on others suggests that the role of that feature is not determined by the nature of the previous trial, as in a feature priming account, but instead is determined by the role played by a given feature on the current trial compared to the previous trial. Huang et al. (2004) argued that this observation is more in line with an episodic retrieval view of priming in singleton search and less so with trial-to-trial adjustments in cognitive control settings. By this view, the attended-to components of the stimulus display are encoded in a single representation, perhaps in the form of an instance as described by Logan (1988), and the retrieval of this representation on a subsequent trial then affects performance. The precise contribution of the previous trial to current performance might occur in 
accordance with the memory principle known as transfer appropriate processing (Morris, Bransford, \& Franks, 1977; see also Neill \& Mathis, 1998; Wood \& Milliken, 1998; Hommel, 1998, for applications to performance tasks). That is, the perceptual characteristics of the current display may cue retrieval of similar prior episodes. Performance will be facilitated to the extent that the processing operations on the current trial match those retrieved as part of the prior processing episode, with strong benefits for perfect matches and weaker benefits or perhaps even costs for partial matches. In this way, an episodic retrieval account seems suitable to explain inter-trial priming effects in singleton search, in particular complex effects involving targetrelevant and target-irrelevant features that might well be difficult to explain by reference to independent and automatic priming of low-level feature representations.

A primary purpose of the present study was to assess the episodic retrieval account of PoP effects. In particular, we were interested in examining predictions such a theory makes that have yet to be subjected to empirical test. It is generally well accepted that episodic memory representations are not limited to the encoding of low-level features and the attentional control settings that lead to the encoding of those features, but rather they include broad task goals of the observer, in line with the transfer-appropriate processing framework (Morris et al., 1977). For example, episodic accounts of negative priming posit that processes involved in withholding a response to a given stimulus dimension on one trial can slow responding when that same stimulus dimension requires a response on the next trial (Hommel, 1998; Tipper, 1985). In this way, it is conceivable that a current search episode cues the retrieval of feature representations, attentional operations, and other higherlevel task-related processes associated with similar prior episodes. If performance in PoP tasks unfolds according to this principle, then a clear empirical prediction is that tasklevel representations ought to mediate PoP effects.

In previous work on PoP, researchers have manipulated the match between previous and current stimulus displays along both target-relevant and target-irrelevant dimensions, but subjects have always performed the same discrimination task from trial to trial, meaning a single task-set is sufficient for good performance in such tasks. By a feature priming account of PoP, a change in selection task from trial to trial, while resulting in a task-switching cost, should not alter the relative contributions of low-level stimulus repeats and alternations from trial to trial, since the same low-level information is used to guide target localization from one trial to the next regardless of the selection task that is to be performed once that target is localized. By an episodic retrieval account, however, the goals of the observer, and therefore the task to be carried out, are bound up in the same episodic memory representation as other low-level target-relevant and target-irrelevant information. By this theory then, task switches should create a partial match between the current and prior processing episodes, thus reducing the degree to which preceding experiences facilitate current action. Thus, by repeating or alternating the selection task from one trial to the next in a predictable manner, it is possible to examine the role of task-set on PoP effects.

To this end, the primary purpose of the present study was to observe how priming of pop-out effects for singleton targets defined by either shape or color are affected by a predictable switch in the response selection task from one trial to the next. A secondary empirical objective was to observe how repetitions versus alternations of a targetirrelevant stimulus feature affected search from trial to trial. In Experiment 1, subjects searched for an odd shape target and then made either a horizontal/vertical judgment about a line inside that shape, or made a ' $\mathrm{T}$ '/ $\mathrm{L}$ ' discrimination to a letter inside the odd shape. In Experiment 2, the color of the stimulus displays either repeated or switched from trial to trial in a random manner, in order to assess the contributions of target-irrelevant information to search performance. In Experiment 3, subjects searched for an odd color target, with the aim of assessing whether priming effects for colordefined targets are more robust to a switch in task. To foreshadow, the results of Experiments 1 and 2 demonstrated that a task-switch reduced the magnitude of PoP effects for shape, while the results of Experiment 3 showed no such modulation of $\mathrm{PoP}$ for color. Experiment 4 demonstrated that a task-switch can indeed reduce PoP effects for color, but this modulation interacted with the relative salience of targets and distractors.

\section{Experiment 1}

The purpose of Experiment 1 was to test how PoP effects are affected by a switch in task. Subjects searched for an odd-shaped target amongst seven homogeneous distractors and reported either the orientation of a line contained within the odd shape or the identity of a letter contained within the odd shape. Priming of pop-out effects (the difference in response time between target feature repetitions and alternations) were analyzed both within and between tasks, to validate the present paradigm's ability to measure such effects within task, and to compare PoP effects within and between tasks. If PoP is mediated solely by low-level feature priming, then a switch in task should not significantly affect the magnitude of PoP. However, if PoP effects are mediated by episodic memory representations created on each trial, a switch in task could create a functionally significant mismatch between the current and prior trial, resulting in significantly smaller PoP effects between tasks relative to within task. 


\section{Method}

Participants

The participants were 20 undergraduates ( 7 male, 13 female) at McMaster University with normal or corrected-to-normal vision. Mean age was 18.2 years. Participants received either course credit or $\$ 5$ for a half hour of participation.

\section{Procedure}

Participants were seated in front of a computer screen and were instructed that upon stimulus onset they were to locate the oddshaped item in the eight-element circular display, which contained one circle and seven squares on half of the trials and one square and seven circles on the other half of the trials. An AABB task switching procedure was used, in which the task to be performed is dictated by where on the screen the search array appears (Rogers \& Monsell, 1995). Half the participants performed a line orientation judgment when the displays appeared in the top two quadrants of the screen and letter discrimination when the displays appeared in the bottom two quadrants of the display, and this assignment of tasks to position was reversed for the other half of the participants. Once a stimulus was responded to, the next search array appeared after a 500-ms blank interval in the location adjacent to the previous array in a clock-wise manner, such that where the next display would appear as well as which task was to be performed was perfectly predictable. The responses for both tasks were mapped to the same keys such that a 'horizontal' or ' $L$ ' response required pressing the ' $Z$ ' key and a 'vertical' or ' $\mathrm{T}$ ' response required pressing the ' $/$ ' key. An example of the procedure is shown in Fig. 1a. Subjects were explicitly told about the predictable task repetitions and alternations and were told to be as fast and accurate as possible when doing both tasks. The first 12 trials were practice trials to familiarize subjects with the tasks and stimulus-response mappings. Response times as well as errors were recorded for an additional 388 trials, for a total of 400 trials. Upon completion, participants were debriefed on the nature of the task and given the opportunity to ask questions.

\section{Stimuli and apparatus}

Subjects were seated approximately $50 \mathrm{~cm}$ away from a computer screen with their index fingers poised over each of the two response keys. Each stimulus display contained eight shapes. Each shape subtended a visual angle of about $1.1^{\circ}$ and the entire stimulus display subtended a visual angle of about $6.5^{\circ}$, with a fixation cross that subtended about $0.6^{\circ}$. For the horizontal/vertical discrimination task, the shapes each contained a single vertical or horizontal line that subtended a visual angle of about $0.8^{\circ}$. For the $\mathrm{T} / \mathrm{L}$ discrimination task, the shapes contained either a single letter $T$ or a single letter $L$ that subtended a visual angle of about $0.6^{\circ}$. Displays appeared in one of four quadrants of the computer screen (although quadrants were not visibly marked on the screen) and remained until response, which was initiated via a key press. The responses 'horizontal' and ' $T$ ' were mapped to the $\mathrm{Z}$ key and the responses 'vertical' and 'L' were mapped to the / key on the keyboard. Stimuli were presented on a 15-inch (c.38.1$\mathrm{cm}$ ) Sony CRT screen. Display elements were created and presented using Presentation Software.

\section{Results}

Only the trials on which correct responses were made were submitted to the response time analysis. As each experimental condition is contingent on the nature of the previous trial type, response times and errors for trials immediately following an error response were also excluded from analysis. All remaining RTs were submitted to an outlier procedure that excluded RTs based on outlier criteria that varied as a function of cell size (see Van Selst \& Jolicoeur, 1994). This analysis eliminated less than $2.5 \%$ of the RTs. Mean RTs were then computed from the remaining observations. These mean RTs and corresponding error rates were submitted to a repeated measures analysis of variance that treated target repetition (target-repeat/targetswitch) and task repetition (task-repeat/task-switch) as within-subject factors. Mean response times are shown in Fig. $1 \mathrm{~b}$ and mean error rates are shown in Table 1.

\section{Response times}

There was a significant main effect of task repetition, with slower responses for task-switch trials $(1,574 \mathrm{~ms})$ than for taskrepeat trials $(1,270 \mathrm{~ms}), F(1,19)=64.62, \mathrm{MSE}=28,705.30$, $p<.0001$. This result is an example of the well-established task switch cost (Allport, Styles, \& Hsieh, 1994; Rogers \& Monsell, 1995). There was also a significant main effect of target repetition, $F(1,19)=54.94$, MSE $=6108.62, p<$ .0001 , with faster responses in the target-repeat $(1,357 \mathrm{~ms})$ than in the target-switch $(1,487 \mathrm{~ms})$ condition. This result is an example of the well-established PoP effect (Maljkovic \& Nakayama, 1994). More important, there was a significant task repetition $\times$ target repetition interaction, $F(1,19)=12.75, \mathrm{MSE}=4723.89, p=.002$. To examine this interaction in further detail, separate analyses of the PoP effect were conducted for the taskrepeat and task-switch conditions. These analyses revealed that the PoP effect was significant in both the task-repeat (184 ms), $t(19)=8.95, p<.0001$, and the task-switch (75 ms), $t(19)=2.91, p=.009$, conditions, but was larger in the task repeat condition. 
Fig. 1 a. A sequence of 3 potential trials in which participants locate and respond to the odd-shape in the display. A task repetition as well as an alternation is depicted. Stimulus displays remained on the screen until a response was made and the next stimulus appeared in the adjacent quadrant after an inter-trial interval of $500 \mathrm{~ms}$. b. Mean reaction time (in ms) for target shape repetitions and alternations as a function of whether the selection task repeated or alternated from trial $n-1$ to trial $n$ a
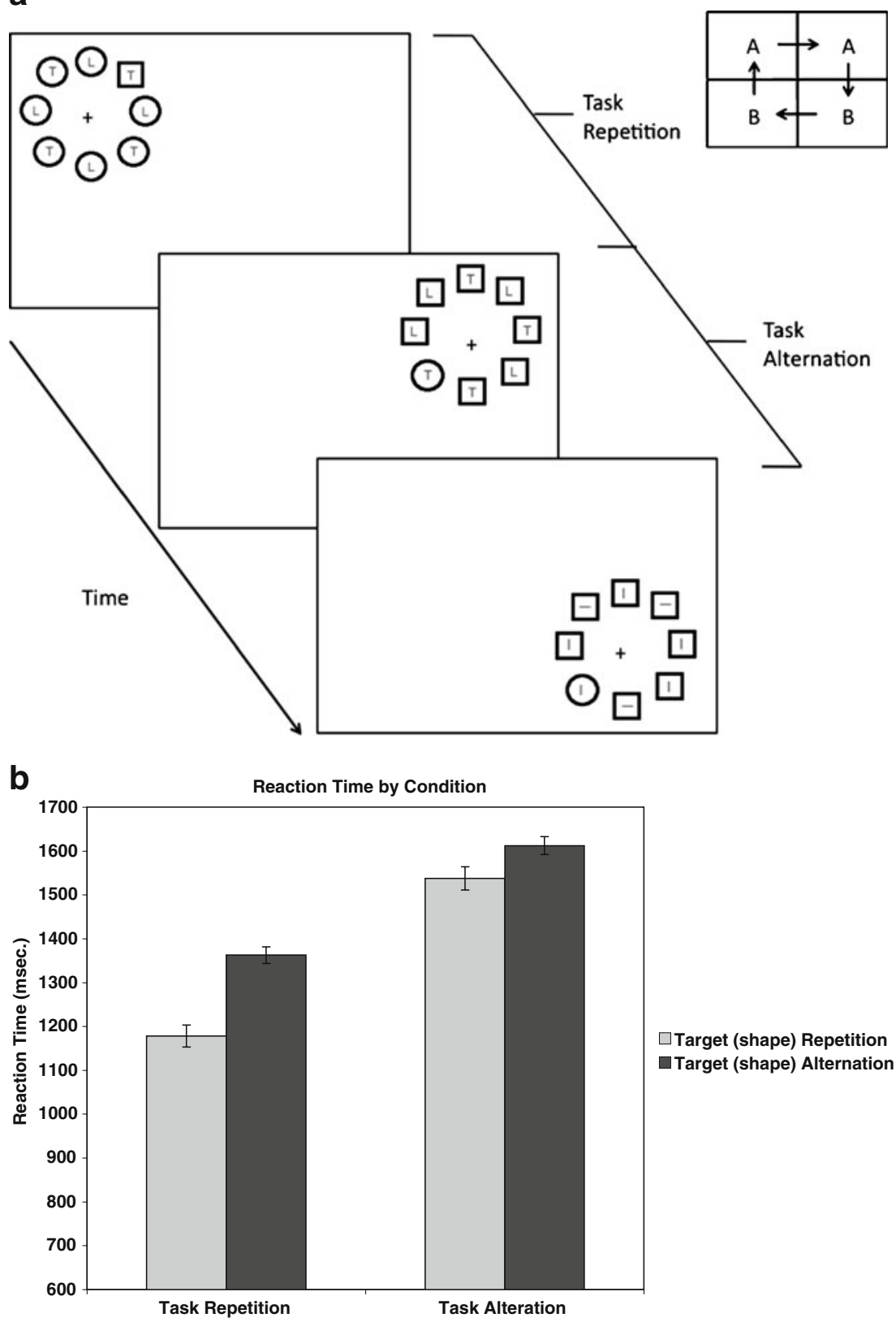

$\square$ Target (shape) Repetition - Target (shape) Alternation
Table 1 Mean error rates and standard deviations (\%) as a function of whether the task repeated or alternated from trial $n-1$ to trial $n$ in Experiment 1

\begin{tabular}{lllll}
\hline & \multicolumn{2}{l}{ Task Repeat } & & \multicolumn{2}{l}{ Task Switch } \\
\cline { 2 - 2 } & $\mathrm{M}$ & & $\mathrm{M}$ & $(\mathrm{SD})$ \\
\hline Target Repeat & $2.38(1.36)$ & & $4.03(1.90)$ \\
Target Switch & $3.18(1.32)$ & & $5.00(1.67)$ \\
\hline
\end{tabular}

Errors

There was a significant main effect of task repetition, with more errors for task-switch $(4.5 \%)$ than for task-repeat trials $(2.8 \%), F(1,19)=12.13, \mathrm{MSE}=4.93, p=.0025$. There was also a significant main effect of target repetition, with significantly more errors for target-switch (4.1\%) than for than target-repeat trials $(3.2 \%), F(1,19)=4.54$, MSE $=$ $3.48, p=.046$. The interaction between task repetition and target repetition was not significant. Overall, the pattern of error rates do not favor a speed-accuracy trade off interpretation of our results. 


\section{Discussion}

The results of Experiment 1 demonstrated that priming of pop-out effects for an odd-shaped target are easily measured with the present task. Furthermore, a clear taskswitching cost was present, with participants responding more slowly on task switches relative to task repetitions. Most important, the significantly smaller PoP effects for task switches than for task repetitions demonstrates that PoP for shape-defined targets is sensitive to switches in task. This result is consistent with the idea that PoP effects are modulated by retrieval of memory episodes in which high-level task representations play a role.

\section{Experiment 2}

The results of Experiment 1 demonstrated that switching tasks between a 'horizontal/vertical' and ' $\mathrm{T}$ or L' judgment significantly reduced PoP effects compared to task repetitions, when the target was defined by shape. This result is in line with a theory in which target shape repetitions were responded to particularly quickly for task repetitions, but not for task alternations, because task alternations require some additional time to resolve the inappropriate taskrelated processes that are bound together with the appropriate target shape information in the same memory episode

The influence of task-irrelevant features on priming effects in pop-out search has also been offered in support of this episodic retrieval explanation of PoP. Huang et al. (2004) had subjects perform a pop-out search task for a target defined by size. It was found that, when target color repeated from one trial to the next, search times were facilitated, but only when the task-relevant feature also repeated. When the taskrelevant feature alternated, irrelevant color repetitions slowed search. These researchers argued that because trial $n-1$ showed qualitatively different effects on current performance depending on the nature of the current trial, low-level feature priming cannot explain the data. In order to examine how a match or mismatch in a target-irrelevant dimension interacts with a task switch, Experiment 2 sought to examine the role of repeating or alternating the task irrelevant color dimension in the present paradigm, while also seeking to replicate the results of Experiment 1.

\section{Method}

Participants

The participants were 20 undergraduates at McMaster University with normal or corrected to-normal vision. Mean age was 20.3 years (6 male, 14 female). Participants received either course credit or $\$ 5$ for a half hour of participation.

\section{Procedure}

The procedure was identical to that of Experiment 1 with the following exception: the color of the shape elements comprising each stimulus display were either red or green in a random fashion, such that the entire display was either red or green on each trial. Thus, the irrelevant color dimension could either repeat or alternate from trial $n-1$ to trial $n$. An example of the procedure is shown in Fig. 2a.

\section{Results}

Only the trials on which correct responses were made were submitted to the response time analysis. Trials immediately following an error response were also excluded from analysis. All remaining RTs were submitted to an outlier procedure (see Van Selst \& Jolicoeur, 1994). Mean RTs were then computed from the remaining observations. These mean RTs and corresponding errors rates were submitted to a repeated measures analysis of variance that treated target repetition (target-repeat/target-switch) as well as irrelevant color repetition (Color-repeat/Color-switch) and task repetition (task-repeat/task-switch) as withinsubject factors. Means of these mean RTs collapsed across participants, are displayed in Fig. $2 \mathrm{~b}$ and error rates are shown in Table 2.

\section{Response times}

There was a significant main effect of task repetition, with slower responses for task-switch trials (1,573 ms) than for task-repeat trials $(1,231 \mathrm{~ms}), F(1,19)=67.61$, MSE $=69,222.4, p<.0001$. There was also a significant main effect of target repetition, $F(1,19)=32.48$, MSE $=$ $21,275.3, p<.0001$, with faster responses in the targetrepeat $(1,336 \mathrm{~ms})$ than in the target-switch $(1,467 \mathrm{~ms})$ condition. More important, there was a significant task repetition $\times$ target repetition interaction, $F(1,19)=10.39$, MSE $=19,966.1, p=.0045$.

To examine the task repetition $\times$ target repetition interaction in further detail, separate analyses of the PoP effect were conducted for the task-repeat and task-switch conditions collapsed across the irrelevant color dimension. These analyses revealed that the PoP effect was significant in the task-repeat $(203 \mathrm{~ms}), t(19)=9.38, p<.0001$, but not the task-switch (59 ms) condition, demonstrating a significantly larger PoP effect in the task repeat condition.

There were no significant effects involving the irrelevant color repetition variable. 
Fig. 2 a. A sequence of 3 potential trials in which participants locate and respond to the odd-shape in the display-the irrelevant color of the display could repeat or alternate from trial $n-1$ to trial $n$. A task repetition as well as an alternation is depicted. Stimulus displays remained on the screen until a response was made and the next stimulus appeared in the adjacent quadrant after an inter-trial interval of $500 \mathrm{~ms}$. b. Mean reaction time (in $\mathrm{ms}$ ) for target shape repetitions and alternations as a function of whether the irrelevant color repeated or alternated and whether the response selection task repeated or alternated

Table 2 Mean error rates and standard deviations (\%) as a function of whether the irrelevant color repeated or alternated and whether the task repeated or alternated from trial $n-1$ to trial $n$ in Experiment 2 a
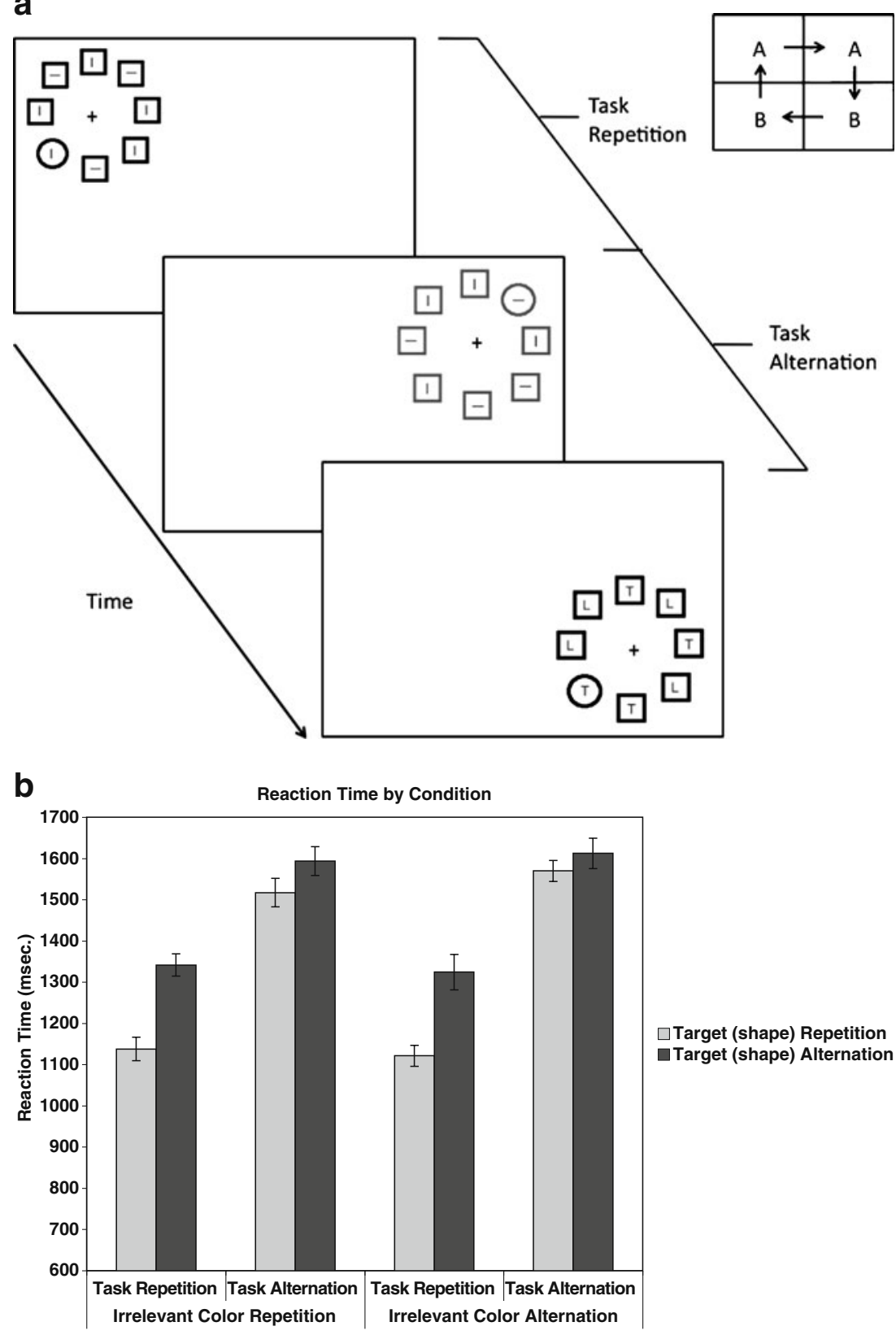

$\square$ Target (shape) Repetition $\square$ Target (shape) Alternation

\begin{tabular}{|c|c|c|c|c|}
\hline & \multicolumn{2}{|c|}{ Irrelevant Color Repetition } & \multicolumn{2}{|c|}{ Irrelevant Color Alternation } \\
\hline & \multicolumn{2}{|l|}{ Task } & \multicolumn{2}{|l|}{ Task } \\
\hline & Repeat & Switch & Repeat & Switch \\
\hline & (SD) & $(\mathrm{SD})$ & $(\mathrm{SD})$ & $(\mathrm{SD})$ \\
\hline Target Repeat & $2.28(2.97)$ & $2.70(2.23)$ & $2.11(1.52)$ & $3.90(2.58)$ \\
\hline Target Switch & $3.11(2.99)$ & $4.66(2.67)$ & $3.56(3.60)$ & $4.12(2.43)$ \\
\hline
\end{tabular}




\section{Errors}

There was a marginally significant main effect of task repetition, with more errors for task-switch $(3.8 \%)$ than for task-repeat trials $(2.8 \%), F(1,19)=3.6$, MSE = $12.84, p=.073$. There was also a significant main effect of target repetition, with significantly more errors for targetswitch $(3.9 \%)$ than target-repeat trials $(2.8 \%), F(1,19)=$ $7.59, \mathrm{MSE}=6.56, p=.0126$. The interaction between task repetition and target repetition was not significant. Overall, the pattern of errors did not favor a speed-accuracy trade-off interpretation of the RT patterns reported above.

\section{Discussion}

One of the purposes of Experiment 2 was to provide a replication of the primary result of Experiment 1, that a shift in the response selection task would modulate PoP effects relative to a task repetition. The results once again show that, overall, PoP was significantly smaller when the response selection task on the previous trial was different than on the current trial. This result provides further support for the hypothesis that episodic retrieval can modulate PoP effects.

A second purpose of Experiment 2 was to extend the findings of Experiment 1 by investigating the role of a taskirrelevant color dimension on inter-trial priming in pop-out search. There was no evidence in the current experiment that irrelevant color repetition from trial $n-1$ to trial $n$ modulated PoP effects, a result that differs somewhat from that reported by Huang et al. (2004), who did report an influence of repetition of an irrelevant color dimension on PoP effects. Although we are uncertain as to why the results from these two studies differ in this respect, there were some methodological differences between their study and ours that may account for the different results. While target color varied randomly (either red or green) on each trial in our experiment, both the target and distractors were all assigned the same color value on a given trial. In other words, all elements in the display were either red or green. In Huang et al. (2004), each item in the display was assigned a random color value on each trial (black or white), such that on each trial there were both black and white elements in the display. Although it is unclear exactly why such a procedural difference would yield different results, it may be that variation of color within a display leads to greater selective attention to the color dimension despite its irrelevance to the task, and in turn attention to the irrelevant color dimension may be critical to observe the result reported by Huang et al. (2004). Put differently, it could be the case that when a target relevant or irrelevant feature varies within a display (e.g., left/right, big/small, black/white) that feature is likely to attract sufficient attention to be encoded in the resulting memory. Although mere speculation at present, this proposal could be tested empirically in future work. In any case, sensitivity of the PoP effect to a switch in task, which was observed here, is consistent with an episodic interpretation of the PoP effect.

\section{Experiment 3}

In the experiments reported so far, the task of the participants was to locate a singleton target shape on each trial. In the original demonstration of PoP by Maljkovic and Nakayama (1994), participants searched for a singleton color target on each trial. The purpose of the present experiment was to examine whether the task switching influence on PoP effects observed in Experiments 1 and 2 for shape singleton targets would also occur for color singleton targets.

\section{Method}

\section{Participants}

The participants were 20 undergraduates at McMaster University with normal or corrected-to-normal vision. Mean age was 19.5 years ( 9 male, 11 female). Participants received either course credit or $\$ 5$ for a half hour of participation.

\section{Procedure}

The procedure was identical to that of Experiment 2 with the following exception: participants searched for an odd colored item in each display instead of an odd-shaped item. The irrelevant stimulus dimension in this experiment was shape, which could repeat or alternate from trial to trial in a random manner. Therefore, each stimulus display consisted of either 8 squares or 8 circles, and the target was either red or green. As before, subjects performed a 'horizontal/vertical' or ' $\mathrm{T}$ or L' judgment on the line or letter contained within the singleton target. An example of the procedure is shown in Fig. 3a.

\section{Results}

Only the trials on which correct responses were made were submitted to the response time analysis. Trials immediately following an error response were also excluded from analysis. All remaining RTs were submitted to an outlier procedure (see Van Selst \& Jolicoeur, 1994). Mean RTs were then computed from the remaining observations. These mean RTs and corresponding error rates were submitted to a repeated 
Fig. 3 a. A sequence of 3 potential trials in which participants locate and respond to the odd-colored item in the display - the irrelevant shape of the stimulus elements could either repeat or alternate from trial $n-1$ to trial $n$. A task repetition as well as an alternation is depicted. Stimulus displays remained on the screen until a response was made and the next stimulus appeared in the adjacent quadrant after an inter-trial interval of $500 \mathrm{~ms}$. b. Mean reaction time (in $\mathrm{ms}$ ) for target color repetitions and alternations as a function of whether the irrelevant shape repeated or alternated and whether the response selection task repeated or alternated
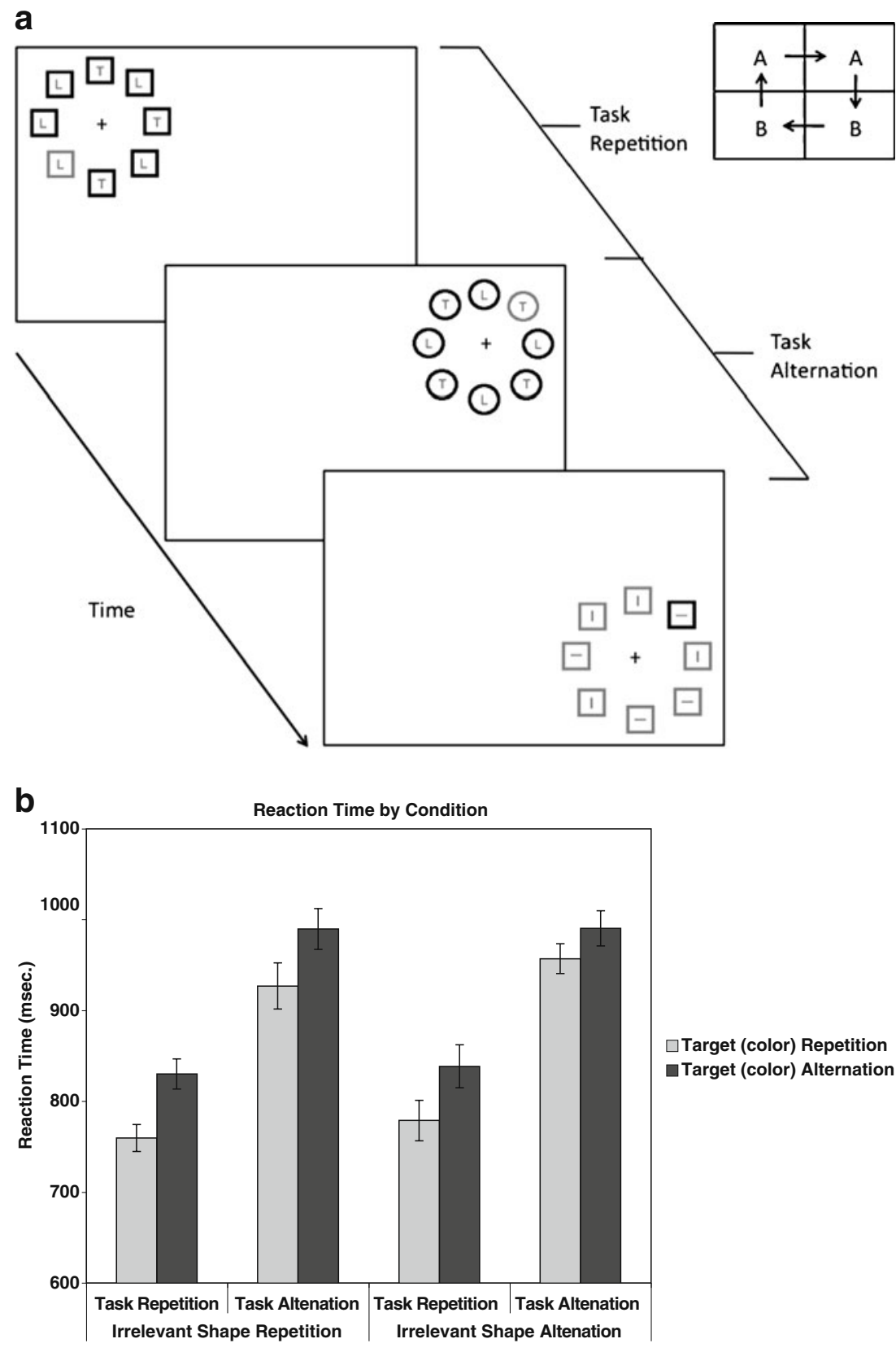

$\square$ Target (color) Repetition $\square$ Target (color) Alternation
Table 3 Mean error rates and standard deviations (\%) as a function of whether the irrelevant shape repeated or alternated and whether the task repeated or alternated from trial $n-1$ to trial $n$ in Experiment 3

\begin{tabular}{|c|c|c|c|c|}
\hline & \multicolumn{2}{|c|}{ Irrelevant Shape Repetition } & \multicolumn{2}{|c|}{ Irrelevant Shape Alternation } \\
\hline & \multicolumn{2}{|l|}{ Task } & \multicolumn{2}{|l|}{ Task } \\
\hline & Repeat & Switch & Repeat & Switch \\
\hline & (SD) & (SD) & (SD) & (SD) \\
\hline Target Repeat & $4.13(4.75)$ & $7.56(4.10)$ & $4.38(2.72)$ & $7.57(4.08)$ \\
\hline Target Switch & $4.39(3.83)$ & $8.60(3.44)$ & $4.85(3.17)$ & $9.27(5.81)$ \\
\hline
\end{tabular}


measures analysis of variance that treated target repetition (target-repeat/target-switch) as well as irrelevant shape repetition (shape-repeat/shape-switch) and task repetition (taskrepeat/task-switch) as within-subject factors. Means of these mean RTs collapsed across participants, are displayed in Fig. $3 b$ and error rates are shown in Table 3.

\section{Response times}

There was a significant main effect of task repetition, with slower responses for task-switch trials $(966 \mathrm{~ms})$ than for task-repeat trials $(801 \mathrm{~ms}), F(1,19)=29.26$, MSE $=$ $36,904.00, p<.0001$. There was also a significant main effect of target repetition, $F(1,19)=11.82$, MSE = $10,852.30, p=.0028$, with faster responses in the targetrepeat $(855 \mathrm{~ms})$ than in the target-switch $(912 \mathrm{~ms})$ condition. Unlike the previous two experiments however, there was no significant task repetition $\times$ target repetition interaction, $F<$ 1. Irrelevant shape repetition did not affect performance either in isolation or in combination with any of the other factors.

\section{Errors}

There was a significant main effect of task repetition, with more errors for task-switch (8.3\%) than for task-repeat trials (4.4\%), $F(1,19)=10.77, \mathrm{MSE}=53.83, p=.0039$. There were no other significant effects observed in the pattern of error rates.

\section{Discussion}

The primary goal of Experiment 3 was to test whether a task switch mediates PoP measured using color singleton targets. Unlike Experiments 1 and 2 when targets were shape singletons, there was no evidence that a switch in task affected PoP effects with color singletons, either when the irrelevant shape dimension repeated or when it switched. Taken literally, the results of Experiments 1-3 might be taken to imply that task switches modulate PoP effects for shape singleton targets but not for color singleton targets, although this is little more than a description of the results. Why shape singletons would have a special status with respect to task switch influences on PoP effects is simply not clear.

An alternative approach to interpreting the results of Experiments 1-3 is to assume that task switching can modulate PoP effects for both shape and color singletons, and for any type of singleton for that matter, but that there are some limiting conditions for observing such an effect. For reasons not yet clear, it may have been the case that the color singleton search task in Experiment 3 approached such a limiting condition, whereas the shape singleton search task in Experiments 1 and 2 was well within the range of conditions under which task switching impacts PoP effects. For example, it could be that singleton salience modulates whether task switching alters PoP effects, with task switching not affecting PoP effects for particularly salient singletons, such as colordefined targets. Indeed, responses in Experiment 3 were approximately $500 \mathrm{~ms}$ faster than those in Experiments 1 and 2 , consistent with the idea that the color singleton targets in Experiment 3 were more salient relative to the distractors than were the shape singleton targets in Experiments 1 and 2. We pursued this issue in Experiment 4 by directly varying relative salience of targets with respect to distractors in a color singleton search task.

\section{Experiment 4}

In this experiment, we examined further whether task switching modulates PoP effects for color singleton targets, and in particular whether such an effect might be modulated by singleton salience. To this end, salience of the color singleton relative to the distracters was manipulated between blocks in the present experiment.

A secondary purpose of Experiment 4 was to address a potential alternative account of the results of Experiments 1 and 2. In particular, note that in the procedure used to this point, participants responded to target stimuli that appeared within the perimeter of target objects, and these target stimuli differed across the two tasks. In one task, the target stimuli were vertical and horizontal lines, whereas in the other task, the target stimuli were the letters ' $\mathrm{T}$ ' and ' $\mathrm{L}$ '. Consequently, a possible criticism of the conclusion that PoP effects varied as a function of task switches in Experiments 1 and 2 is that perceptual mismatches between the target stimuli for the two tasks rather than the task switch itself may have been responsible for the effects observed. To address this issue, we used identical target stimuli for the two tasks in Experiment 4. An additional criticism that might be leveled against the results of Experiments 1 and 2 is that responses could require participants to first locate the target object and then to re-focus attention to the target stimulus within the target object. In contrast, the procedure often used to measure PoP effects (e.g., Bravo \& Nakayama, 19921; Maljkovic \& Nakayama, 1994) requires participants to respond to a property of the target object (e.g., which side of a shape is missing a corner), rather than to a target stimulus within the target object. To address this issue, we had participants respond to a property of the target object in Experiment 4, with participants indicating whether a gap in the perimeter of a target object was on the left or right for one task, or on the top or bottom for the other task. 


\section{Method}

Participants

The participants were 32 undergraduates ( 4 male, 28 female) at McMaster University with normal or corrected to normal vision. Mean age was 18.9 years. Participants received either course credit or $\$ 5$ for a half hour of participation.

\section{Procedure}

Participants were seated in front of a computer screen and were instructed that upon stimulus onset they were to locate the odd-colored item in the eight-element circular display, and make either a top/bottom or left/right decision about the gap in the odd colored item, depending on the quadrant of the screen in which the stimulus display appeared. An AABB task switching procedure was used, in which the task to be performed was dictated by where on the screen the search array appeared (Rogers \& Monsell, 1995). Half the participants performed a top/bottom judgment when the displays appeared in the top two quadrants of the screen and a left/right discrimination when the displays appeared in the bottom two quadrants of the display, and this assignment of tasks to position was reversed for the other half of the participants. Once a stimulus was responded to, the next search array appeared after a 500-ms blank interval, and in the location adjacent to the previous array in a clock-wise direction. As such, the position of the next display as well as which task was to be performed was perfectly predictable. The responses for both tasks were mapped to the same keys such that a 'left' or 'top' response required pressing the ' $\mathrm{Z}$ ' key and a 'right' or 'bottom' response required pressing the '/' key. Subjects were explicitly told about the predictable task repetitions and alternations and were asked to be as fast and accurate as possible when doing both tasks.

The experiment was divided into two blocks of trials. For one block, the relative salience of the target and distracters was similar to that in Experiment 3, with green singleton targets surrounded by red distracters or vice versa. For the other block, the relative salience of target and distracters was manipulated such that a green target would be surrounded by turquoise distracters or vice versa. The order of the two blocks was randomly counterbalanced between participants. The first 12 trials of each block were practice trials to familiarize subjects with the tasks and stimulus-response mappings. Response times as well as errors were recorded for an additional 388 trials, for a total of 400 trials per block. Upon completion, participants were debriefed on the nature of the task and given the opportunity to ask questions. An example of the procedure is shown in Fig. 4a.
Stimuli and apparatus

Subjects were seated approximately $50 \mathrm{~cm}$ away from a computer screen with their index fingers poised over each of the two response keys. Each stimulus display contained eight squares with a gap in either the top or the bottom and in either the left or the right side. Each shape subtended a visual angle of about $1.1^{\circ}$ and the entire stimulus display subtended a visual angle of about $6.5^{\circ}$, with a fixation cross that subtended about $0.6^{\circ}$. Displays appeared in one of four quadrants of the computer screen (although quadrants were not visibly marked on the screen) and remained until response, which was initiated via a key press. The responses 'left' and 'top' were mapped to the ' $Z$ ' key and the responses 'right' and 'bottom' were mapped to the '/ key on the keyboard. Stimuli were presented on a 15 -inch $(\mathrm{c} .38 .1-\mathrm{cm})$ Sony CRT screen. For the high-salience condition, target and distracter elements were red and green whereas in the low-salience condition, target and distractor elements were turquoise and green. Display elements were created and presented using Presentation Software.

\section{Results}

Only the trials on which correct responses were made were submitted to the response time analysis. Trials immediately following an error response were also excluded from analysis. All remaining RTs were submitted to an outlier procedure (see Van Selst \& Jolicoeur, 1994). Mean RTs were then computed from the remaining observations. These mean RTs and corresponding errors rates were submitted to a repeated measures analysis of variance that treated target repetition (target-repeat/target-switch), task repetition (task-repeat/task-switch) and block (highsalience/low-salience) as within-subject factors; block order was treated as a between subjects factor. Mean response times collapsed across participants are shown in Fig. $4 \mathrm{~b}$ and error rates are shown in Table 4.

\section{Response times}

There was a main effect of salience, with RTs being faster in the high-salience condition $(1,076 \mathrm{~ms})$ than the lowsalience condition $(1,132 \mathrm{~ms}), F(1,30)=4.43, \mathrm{MSE}=$ $45,389, p=.0438$. There was a significant main effect of task repetition, with slower responses for task-switch trials $(1,277 \mathrm{~ms})$ than for task-repeat trials $(932 \mathrm{~ms}), F(1,30)=190$, $\mathrm{MSE}=38,755.70, p<.0001$. There was also a significant main effect of target repetition, $F(1,30)=42.47$, MSE $=$ $9,615.96, p<.0001$, with faster responses in the target-repeat $(1,063 \mathrm{~ms})$ than in the target-switch $(1,145 \mathrm{~ms})$ condition. 
Fig. 4 a. A sequence of 3 potential trials in which participants locate and respond to the odd-colored item in the display - target and distractor salience was manipulated between conditions. A task repetition as well as an alternation is depicted. Stimulus displays remained on the screen until a response was made and the next stimulus appeared in the adjacent quadrant after an inter-trial interval of $500 \mathrm{~ms}$. b. Mean reaction time (in $\mathrm{ms}$ ) for target color repetitions and alternations as a function of whether the selection task repeated or alternated, from trial $n-1$ to trial $n$, and whether the relative salience of target and distractors was high or low a
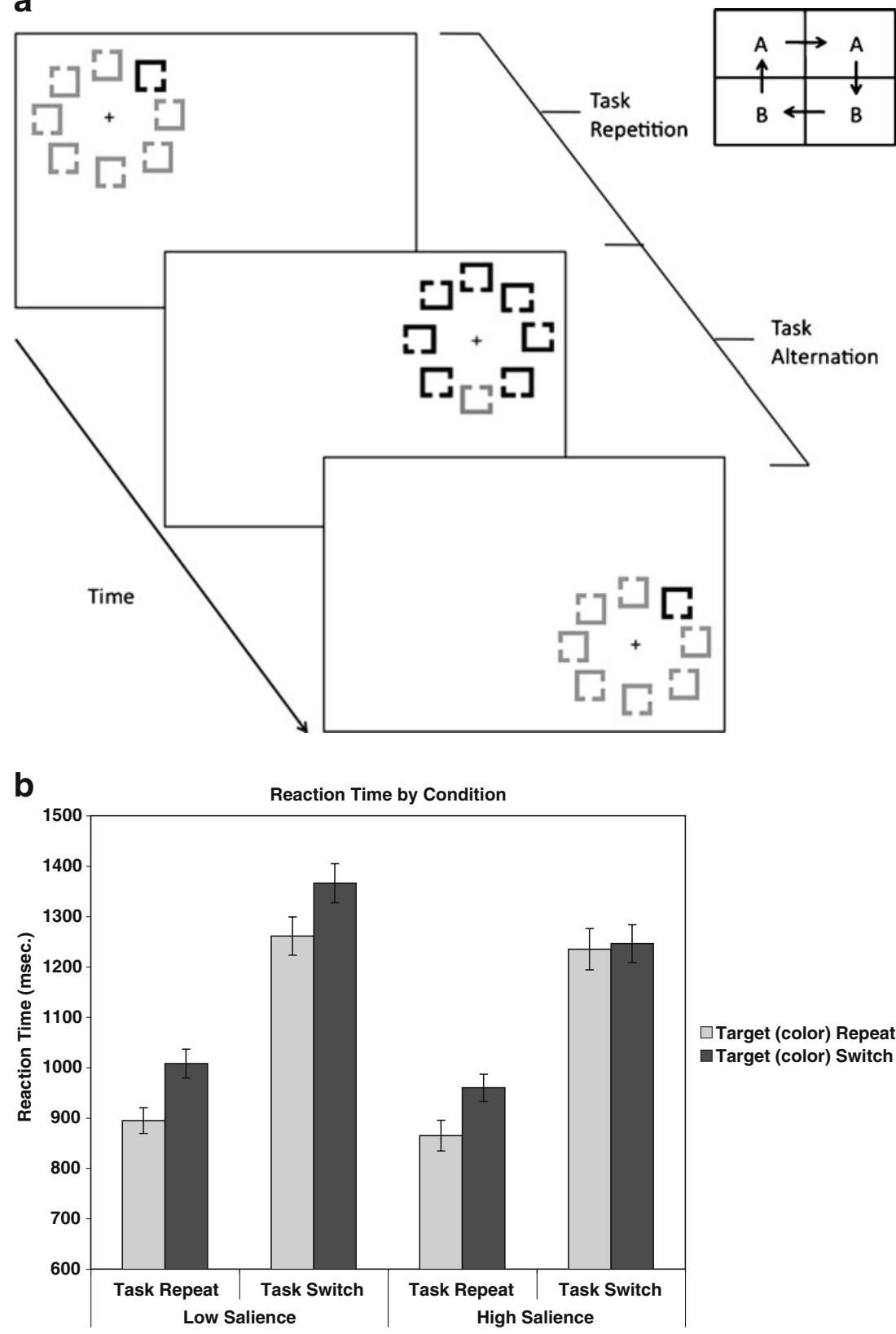

$\square$ Target (color) Repeat Target (color) Switch
Table 4 Mean error rates and standard deviations (\%) for the high and low-salience conditions as a function of whether the task repeated or alternated from trial $n-1$ to trial $n$ in Experiment 4

\begin{tabular}{|c|c|c|c|c|}
\hline & \multicolumn{2}{|l|}{ Low-Salience } & \multicolumn{2}{|l|}{ High-Salience } \\
\hline & \multicolumn{2}{|l|}{ Task } & \multicolumn{2}{|l|}{ Task } \\
\hline & Repeat & Switch & Repeat & Switch \\
\hline & $\mathrm{M} \quad(\mathrm{SD})$ & (SD) & (SD) & (SD) \\
\hline Target Repeat & $2.07(3.05)$ & $5.20(3.01)$ & $2.99(3.66)$ & $6.38(4.11)$ \\
\hline Target Switch & $2.91(3.74)$ & $5.19(4.21)$ & $3.25(3.05)$ & $5.56(3.93)$ \\
\hline
\end{tabular}


The 3-way interaction between target, task, and salience, was significant, $F(1,30)=5.25, \mathrm{MSE}=4,333.70, p=$ .0269 . To interpret this interaction, separate 2 (target repeat/ switch) $\times 2$ (task repeat/switch) ANOVAs were conducted to assess how PoP was affected by a task switch in the high- and low-salience conditions. For the low-salience condition, there was no significant 2-way interaction between task and target, $\mathrm{F}<1$. PoP effects, therefore, were not affected by a switch in task in this condition. For the high-salience condition, there was a significant 2-way interaction between task and target, $F(1,31)=9.65$, MSE $=$ $5,950.06, p=.004$. Thus, the significant 3 -way interaction is being driven by a significant modulation of priming of popout across task in the high-salience, but not the low-salience condition. To confirm this interpretation, separate analyses of the PoP effect were conducted for the task-repeat and task-switch conditions for the high-salience condition. These analyses revealed that the PoP effect was significant in the task-repeat condition (95 ms), $t(31)=5.12, p<.0001$, but not the task-switch $(11 \mathrm{~ms})$ condition.

\section{Errors}

The only significant effect in this analysis was the main effect of task repetition, with more errors committed for task-switch trials $(5.6 \%)$ than for task-repeat trials $(2.8 \%)$, $F(1,31)=52.87, \mathrm{MSE}=9.15, p<.0001$.

\section{Discussion}

The results of Experiment 4 demonstrate clearly that PoP effects for color singleton targets can be modulated by task switches. In the high-salience condition, the PoP effect was larger for task repeats than for task alternations. As such, it seems clear that the task switch modulation of the PoP effect observed in Experiments 1 and 2 is not idiosyncratic to shape singleton targets. At the same time, the fact that this effect occurred for the high-salience condition and not for the low-salience condition undermines any simple notion that the different results for shape and color singleton targets across Experiments 1-3 was a result of salience differences between the shape and color singleton targets in these experiments. Indeed, the high-salience condition of the present experiment used color pop-out targets that were very similar to those used in Experiment 3, and yet task switches modulated PoP effects here but not in Experiment 3. These results highlight the fact that factors other than singleton salience must dictate the extent to which task switching modulates PoP effects. This issue is addressed in more detail in the General discussion.

An additional implication of the results of this experiment is that task switch modulations of PoP effects do not require perceptual mismatches between the targets of the two tasks. Whereas in Experiments 1-3 participants responded to different target stimuli in the two tasks, the target stimuli in the two tasks were identical in the present experiment. In addition, the task switch modulation of color PoP observed in the high-salience condition here occurred in a task much like that used originally by Bravo and Nakayama (see also Maljkovic \& Nakayama, 1994), in that participants responded to a property of the target object itself rather than to a target stimulus that appeared within the target object.

A final concern that might be expressed about this experiment is that we have no basis for being sure that the targets in the low-salience condition truly allowed pop-out search, as the target and distractor colors were quite similar in this condition. Our response to this concern is that, indeed, the low-salience targets may not have popped out to the same extent as the high-salience targets (although note that the mean RTs differed by only about $50 \mathrm{~ms}$ across these two conditions), but the key result here is the result in the high-salience condition. In particular, for high-salience singleton color targets, a switch in task did modulate the PoP effect, a result that is difficult to accommodate without reference to the involvement of higher order task level aspects of representation.

\section{General discussion}

The primary goal of the experiments reported here was to examine whether a higher order switch in selection task from trial $n-1$ to trial $n$ would affect the priming of pop-out effect in visual search. Experiments 1 and 2 demonstrated that a switch in selection task significantly reduced the magnitude of the PoP effect, when the target was defined as the odd-shaped item in the display. Experiment 3 failed to show such modulation when the target was defined as the odd-colored item in the display. Experiment 4 demonstrated that PoP for color can indeed be modulated by a switch in selection task from trial $n-1$ to $n$, in this case when the relative salience of targets with respect to distractors was high. Although the role of stimulus salience in this effect is unclear and will be an issue for future study, the primary contribution of the present work is to show that the memory representations that drive $\mathrm{PoP}$ can include higher-order information as well as low-level perceptual information.

Maljkovic and Nakayama's initial (1994, 1996, 2000; see also Bravo \& Nakayama, 1992) explanation of PoP proposed that such effects result from the short-term persistence of a memory representation involving the target-defining feature. Clearly, the results of the present experiments suggest that more than the target-defining feature is involved in the memory representation that 
supports PoP effects. Although other studies have also implicated memory representations involving more than just target-defining information (Huang et al., 2004; Lee et al., 2009), to our knowledge ours is the first study to implicate higher-level task-related processes in the memory representations that support PoP.

Lee et al. (2009) have forwarded an account of PoP that describes how multiple pieces of information can be represented from one trial to the next, and subsequently affect performance. These researchers have argued that independent activation/suppression of 'feature weights' from one trial to the next are what create memory representations that persist across trials. In this view, perceptual properties of a target stimulus are independently represented in memory for a short time. The way in which these weights are argued to be modulated from one trial to the next is via cognitive control operations that activate or suppress certain features of the stimulus display in accord with the demands of the task (goals of the observer). Much like the memory trace posited by Maljkovic and Nakayama (1994), these representations of 'feature weights' persist for a short time, allowing search time savings if a subsequent trial requires the same assignment of feature weights, or costs if different or opposite weights are required. A central tenet of feature modulation theory is that different features are activated and suppressed in an independent manner. That is, a memory trace could include the activation of one feature, say, 'red', and the activation of a separate feature, say, 'large', that would provide search time savings if the next trial included target features that were either 'red' or 'large'. Even more savings in search times would be observed if the next trial included target features that were both 'red' and 'large', and thus additivity of these factors from trial $n-1$ to trial $n$ would be a clear demonstration of the independence of the feature representations of 'red' and 'large'. Furthermore, this theoretical interpretation of PoP posits that only low-level, or perceptual information gets represented from one trial to the next. That is, there is no obvious way in which feature gains can be adjusted for non-perceptual information. In summary, the feature gain modulation account of PoP assumes independent representation of low-level perceptual attributes, the memory of which persists from one trial to the next.

An alternative account of PoP that also allows for a memory influence from more than one stimulus dimension assumes that multiple stimulus dimensions are bound together in memory rather than represented independently. Huang et al. (2004) argued specifically that such bound representations, referred to as memory episodes, might underlie PoP effects in singleton search. Like feature gain modulation theory, an episodic theory predicts that performance on trial $n$ should be affected by whether or not trial $\mathrm{n}$ shares perceptual properties with trial $n-1$. Unlike feature gain modulation theory, an episodic theory predicts interactions involving repetition/alternation of separate features. For example, repeating one feature of a stimulus, but alternating another, might well slow search time relative to a condition in which both stimulus features alternate (see Hommel, 1998). Another theoretical distinction between feature gain modulation and episodic theories is that episodic representations can in principle include higher level (non-perceptual) information as well as low level (perceptual) information, whereas it is not obvious how task-related information can be incorporated into a feature gain modulation account of PoP. With respect to the results of the present experiments, target and task variables clearly can affect performance in a non-additive manner; that is, in at least some contexts task and target repetition interact to produce significantly smaller PoP effects for task switches than for task repetitions. Such results appear easier to accommodate within an episodic retrieval framework than within a feature gain modulation framework for explaining PoP.

Lee et al. (2009) did offer a way in which feature modulation theory can account for non-additive effects of target relevant and irrelevant information in pop-out search. In particular, they stated that the results of Huang et al. (2004) can be accounted for within a feature modulation framework if we assume that the features that represent an object are bound together in memory, with independent weights set for each feature. To the extent that the encoded 'object' changes from one trial to the next, performance would be expected to suffer.

Put in these terms, the feature modulation account of PoP effects has much in common with other theories that have invoked the integration of features into object representations to explain inter-trial priming effects. Kahneman, Treisman and Gibbs (1992) showed that the ability of participants to respond to the second presentation of a letter was significantly diminished when that letter appeared within a different object from one exposure to the next. They argued that features of objects are bound together in memory, creating what they referred to as 'object files', which are created and retrieved on each trial. The only real distinction to be made between the explanation of Lee et al. (2009) with regards to bound objects and the concept of object files as offered by Kahneman et al. (1992) is that Kahneman et al. went to some lengths to demonstrate that object files can be retrieved and updated upon onset of a subsequent stimulus display, rather than simply persisting passively for some limited amount of time.

The notion of bound representations of feature weights, or of object files, holds a lot of explanatory power in accounting for interactions among low-level perceptual attributes in a pop-out search task. However, the present 
results demonstrate an interaction involving both low-level stimulus attributes and higher-level, non-perceptual information. With this in mind, it is noteworthy that Hommel (1998) extended the concept of object files to include the goals and the behavior of participants. By this view, perceptual as well as non-perceptual attributes of a given trial are bound up in a single representation that Hommel referred to as an 'event file'. This extended episodic framework allows for, and indeed predicts, that switching task in the present study should reduce the facilitation that occurs for target repetitions from trial $n-1$ to $n$.

Although it is clear that we have found conditions under which higher-level influences can be observed on the PoP effect, we have also discovered conditions under which no such influences can be observed. For example, PoP was modulated by task switches for the high-salience condition of Experiment 4, but not in Experiment 3 when the same target and distractor colors were used, and only for targets defined by shape across Experiments 1-3. It is therefore clear that there are limiting conditions for observing interactions among higher-level and lower-level stimulus attributes in a singleton search task. While the pursuit of these limiting conditions is an issue for further study, two observations can be made here. First, methodological changes between Experiments 1-3 and Experiment 4 make it difficult to postulate a single process or stimulus attribute that can adequately account for the discrepancies in our results. Specifically, recall that, in Experiments 1-3, participants had to re-focus attention within the target object once it was localized in order to respond, whereas in Experiment 4, no such re-focusing was required. Second, it is clear that neither the relative salience of targets with respect to distractors nor the target-defining feature (shape/ color) alone can delineate these limiting conditions.

In light of these observations, a full account of the results reported here might ultimately have to take into account strategic influences on task-related processes. In particular, it seems possible that participants may approach the search task in functionally different ways in different contexts. For example, on a trial that involves a task switch one could first engage the appropriate task-set for that trial and then localize the target, or one could localize the target and then engage the appropriate task-set. From an episodic perspective, the cues that drive retrieval of prior episodes would be entirely different in these two cases and, therefore, for example, the former strategy might be less likely to demonstrate a PoP effect in the context of a task switch. Admittedly, such an account is speculative, as it is not yet clear which particular combinations of task variables would lead one strategy to dominate over the other, but it does offer a starting point for further empirical work.

In summary, there exists debate in the literature with respect to the nature of the memory mechanism underlying the priming of pop-out effect. Some researchers argue that the existing data are best explained via reference to independent activation and suppression of feature weights that persist from one trial to the next (Lee et al., 2009). Others interpret the data as being best explained within an episodic retrieval framework, in which perceptual and nonperceptual information is represented in a non-independent manner from one trial to the next (Huang et al., 2004). The results of the present study demonstrate that, under some conditions, the effect of higher-level, non-perceptual information can affect the PoP effect. Moreover, this non-perceptual information interacts with low-level target-defining information, suggesting that these attributes are bound together in a non-independent manner in memory. Although it is clear that much more work must be done to better elucidate the conditions under which higher-level influences on PoP can be observed, making use of episodic retrieval theories to make predictions about the nature of PoP effects seems a productive endeavor in light of the present work.

Acknowledgements This research was supported and funded by an NSERC Discovery Grant. We thank Liqiang Huang and an anonymous reviewer for their helpful comments and suggestions on an earlier version of this manuscript.

\section{References}

Allport, D. A., Styles, E. A., \& Hsieh, S. (1994). Shifting intentional set: Exploring The dynamic control of tasks: Umlita, Carlo (Ed).

Bravo, M., \& Nakayama, K. (1992). The role of attention in different visual search tasks. Perception \& Psychophysics, 51, 465-472.

Hillstrom, A. P. (2000). Repetition effects in visual search. Perception \& Psychophysics, 62(4), 800-817.

Hommel, B. (1998). Event files: Evidence for automatic integration of stimulus-response episodes. Visual Cognition, 5(12), 183-216.

Huang, L., Holcombe, A. O., \& Pashler, H. (2004). Repetition priming in visual search: Episodic retrieval, not feature priming. Memory \& Cognition, 32(1), 12-20.

Kahneman, D., Treisman, A., \& Gibbs, B. (1992). The reviewing of object files: Object-specific integration of information. Cognitive Psychology, 24, 175-219.

Kristjansson, A. (2006). Simultaneous priming along multiple feature dimensions in a visual search task. Vision Research, 46, 2554 2570 .

Lee, H., Mozer, M. C., \& Vecera, S. P. (2009). Mechanisms of priming of pop-out: Stored representations or feature-gain modulations? Attention Perception \& Psychophysics, 71(5), 1059-1071.

Logan, G. D. (1988). Toward an instance theory of Automatization. Psychological Review, 95(4), 492-527.

McBride, J., Leonards, U., \& Gilchrist, I. D. (2009). Flexible target representations underlie repetition priming in visual search. Visual Cognition, 17, 655-678.

Maljkovic, V., \& Nakayama, K. (1994). Priming of pop-out: I. Role of features. Memory \& Cognition, 22(6), 657-672. 
Maljkovic, V., \& Nakayama, K. (1996). Priming of pop-out: II. The role of position. Perception \& Psychophysics, 58(7), 977-991.

Maljkovic, V., \& Nakayama, K. (2000). Priming of pop-out: III. A short-term implicit memory system beneficial for rapid target selection. Visual Cognition, 7(5), 571-595.

Morris, C. D., Bransford, J. D., \& Franks, J. J. (1977). Levels of processing versus transfer appropriate processing. Journal of Verbal Learning and Verbal Behavior, 16, 519-533.

Neill, W. T., \& Mathis, K. M. (1998). Transfer-inappropriate processing: Negative priming and related phenomena. The Psychology of Learning and Motivation, 38, 1-44.

Olivers, C. N. L., \& Humphreys, G. W. (2003). Attentional guidance by salient feature singletons depends on intertribal contingencies. Journal of Experimental Psychology: Human Perception and Performance, 29, 650-657.

Rogers, R. D., \& Monsell, S. (1995). Costs of a predictable switch between simple cognitive tasks. Journal of Experimental Psychology: General, 124, 207-231.

Treisman, A. M., \& Gelade, G. (1980). A feature-integration theory of attention. Cognitive Psychology, 12, 97-136.
Tipper, S. P. (1985). The negative priming effect: Inhibitory priming by ignored objects. The Quarterly Journal of Experimental Psychology, 37, 571-590.

Van Selst, M., \& Jolicoeur, P. (1994). A solution to the effect of sample size on outlier elimination. The Quarterly Journal of Experimental Psychology, 47(3), 631-650.

Wolfe, J. M. (2003a). Changing your mind: On the contributions of top-down and Bottom-up guidance in visual search for feature singletons. Journal of Experimental Psychology: Human Perception and Performance, 29(2), 483-502.

Wolfe, J. M. (2003b). Moving towards solutions to some enduring controversies in visual search. TRENDS in Cognitive Science, 7 (2), 70-76.

Wolfe, J. M., Butcher, S. J., Lee, C., \& Hoyle, M. (2003). Changing your mind: On the Contributions of top-down and bottom-up guidance in visual search for feature singletons. Journal of Experimental Psychology: Human Perception and Performance, 29, 483-502.

Wood, T., \& Milliken, B. (1998). Negative priming without ignoring. Psychonomic Bulletin \& Review, 5, 470-475. 